\title{
Expansion for the Moments of a Nonlinear Stochastic Model
}

\author{
A. N. Drozdov* and M. Morillo \\ Física Teórica, Universidad de Sevilla, Apartado Correos 1065, Sevilla 41080, Spain
}

(Received 4 June 1996)

\begin{abstract}
We present a procedure to systematically evaluate all the moments of the Fokker-Planck equation by expanding them in a power series in a given function of $t$. The expansion coefficients are easily determined in terms of algebraic recursion relations. Applications to a linear Fokker-Planck equation, as well as to a truly nonlinear mean-field model, whose drift coefficient exhibits a functional dependence on the distribution function, show this formalism to be advantageous over the standard time series expansion of the moments which is shown to be rather impractical. [S0031-9007(96)01444-5]
\end{abstract}

PACS numbers: 05.40.+j, 02.50.Ey, 02.50.-r

There is a growing literature on the study of fluctuations in nonlinear systems far from equilibrium. Here, we will deal with one of the extensively studied models, namely, the so-called mean-field model, which describes a system of infinitely many nonlinear coupled oscillators in the presence of an external white noise. It was originally introduced by Kometani and Shimizu to study self-organization processes in biological systems such as muscle contraction [1]. A more complete statisticalmechanical treatment given later by Desai and Zwanzig [2] and by Dawson [3] pointed out its relation with the Weiss-Ising model. These authors also showed that the combined effect of thermal noise and mean-field interaction gives rise to a truly nonlinear Fokker-Planck equation for the probability density associated with the order parameter. The equation reads

$$
\begin{gathered}
\partial_{t} P(x, t)=\partial_{x}\left[x^{3}+(\theta-1) x-\theta\langle x(t)\rangle\right. \\
\left.+D \partial_{x}\right] P(x, t),
\end{gathered}
$$

where the drift force depends upon the state of the system through the average

$$
\langle x(t)\rangle=\int_{-\infty}^{\infty} d x P(x, t) x .
$$

An important feature of the above model is that it shows a genuine order-disorder phase transition. Because of the nonlinear term $\theta\langle x(t)\rangle \partial_{x} P(x, t)$, there exists a critical line in the parameters space such that when $D$ and/or $\theta$ are varied across this line the equilibrium distribution bifurcates $[2,4]$. Below the critical line the equilibrium is unique regardless of the initial conditions. Above the critical line there are three equilibrium solutions, one unstable, and two stable ones, and, as $t$ goes to infinity, the system approaches one of the equilibria depending upon the value of $\langle x(0)\rangle$, or, in other words, upon the initial preparation of the system.

It is not hard to construct an approximate solution of a Fokker-Planck equation which is valid in the short time limit, e.g., by using an operator decoupling technique [5]. Exact results are also available in the long time limit when the system approaches equilibrium [4]. Beyond the above limits, a number of approximate methods have been introduced in order to solve Eq. (1) numerically [2,6,7] (see also a collection of references in Ref. [7]). However, in spite of the advances of even the fastest algorithms and computers, the calculation of the dynamical properties of nonlinear systems for all times is known to be a difficult problem in statistical mechanics.

In this Letter we outline a method for systematically calculating the moments of the Fokker-Planck equation in a straightforward analytical way. To keep the presentation simple we have avoided considering systems with many degrees of freedom or the case of other nonlinearities in Eq. (1). The method explained below can be easily modified to cover those cases. The key point of the method is the same as in a power series expansion formalism which has been used so far in studying linear systems [8-12] (for a recent review see Ref. [13]). One of the underlying ideas of this formalism is representing the propagator as a power series in $t$. In the simplest realization it reads

$$
\begin{aligned}
P(x, t \mid y) & =e^{t L(x)} \delta(x-y) \\
& =e^{t L^{+}(y)} \delta(x-y)=\sum_{m=0}^{\infty} P_{m} \frac{t^{m}}{m !}, \\
P_{m} & =L^{m} \delta(x-y)=\left(L^{+}\right)^{m} \delta(x-y),
\end{aligned}
$$

where $L$ and $L^{+}$are the forward and backward FokkerPlanck operators which are assumed to be linear and time independent and act on the $x$ and the $y$ variables, respectively. Applying Eq. (3) to the average

$$
\langle g(x)\rangle=\int_{-\infty}^{\infty} d x P(x, t \mid y) g(x),
$$

one immediately obtains

$$
\langle g(x)\rangle=\sum_{m=0}^{\infty} g_{m}(y) \frac{t^{m}}{m !}, \quad g_{m}(y)=\left(L^{+}\right)^{m} g(y) .
$$

Equation (5) constitutes the main result of a recent paper by Weiss and Gitterman [11]. Unfortunately, these authors have only illustrated the feasibility of their 
expansion in linear exactly solvable models. Little is known about the convergence properties of the series, although our experience with similar problems $[10,12,13]$ suggests that its utility is restricted to short times. Indeed, the calculations we performed with Eq. (5) on model systems show that the method is accurate in this limit if one truncates the series at high enough order $m=M$. With increasing $t$, however, the error begins to grow very rapidly, and beyond some $t_{\max }$ which is usually noticeably smaller than unity, the expansion fails to produce convergent results with a finite number of terms taken into account. The reason for this is that any finite truncation of the series in Eq. (5) tends to plus or minus infinity depending on the sign of $g_{M}$. The same is true, in one sense or another, to many other different Taylor-like expansions available in the literature [8-10].

Drozdov has recently presented an alternative power series expansion of the propagator which is free of the above drawback [12,13]. A few terms of this expansion are sufficient to attain an accurate description of the dynamics in the whole time domain. Certainly, the expansion coefficients of this more rapidly convergent power series representation are more difficult to evaluate than those of Eq. (5), but it is not so highly dramatic in many situations of practical interest [13]. Unfortunately, the same is not true for nonlinear problems. Although it is not difficult to formally apply the results of $[12,13]$ to Eq. (1), the calculation of the expansion coefficients becomes very arduous in this case.

Our aim is to develop an alternative power series expansion for the moments which is convergent over a broader range of $t$, and easily applicable to both linear and nonlinear problems. As the treatment outlined below is fairly straightforward, we will omit the details of the calculations and show only the main results. For the sake of generality we consider a nonlinear Fokker-Planck equation of the form

$$
\partial_{t} P(x, t)=-\partial_{x}\left[G(x)+F(x)\langle x(t)\rangle-\partial_{x} D(x)\right] P(x, t),
$$

where power series expansions for the coefficients

$$
R(x)=\sum_{i=0}^{\infty} R_{i} x^{i}, \quad R=G, F, D,
$$

are assumed to exist. Multiplying both sides of Eq. (6) with $x^{k}$ and integrating over $x$, one arrives at the following hierarchy of first order coupled differential equations for the moments:

$$
\begin{aligned}
\partial_{t}\left\langle x^{k}\right\rangle=k\left[\sum_{i=0}^{\infty}\left(G_{i}+F_{i}\langle x\rangle\right)\left\langle x^{k+i-1}\right\rangle\right. \\
\left.+(k-1) D_{i}\left\langle x^{k+i-2}\right\rangle\right], \\
k=1,2, \ldots,
\end{aligned}
$$

with the initial conditions

$$
\left\langle x^{k}(0)\right\rangle=\int_{-\infty}^{\infty} d x P(x, 0) x^{k} .
$$

The above equations can be exactly solved by expanding $\left\langle x^{k}\right\rangle$ in powers of a given function of $t$,

$$
\left\langle x^{k}(t)\right\rangle=\sum_{m=0}^{\infty} x_{k, m} \tau^{m}(t)
$$

Here, we restrict ourselves to the same function $\tau(t)$ as in Refs. [12,13],

$$
\tau=\frac{1}{\omega}\left(1-e^{-\omega t}\right),
$$

though a generalization to an arbitrary dependence $\tau$ of $t$ is also possible. This particular time dependence has been chosen as it is associated with the width of the fluctuations in a linear problem, and so it is expected to provide one with a reasonable time scale of the fluctuations in a general case. From a computational point of view, the most appealing feature of expanding in $\tau$ in place of $t$ is perhaps that with $\omega>0$ the time transformation maps the singular point $t=\infty$ to finite $\tau=1 / \omega$. Moreover, the frequency $\omega$, which is a free parameter, can be chosen such that the convergence of the series in Eq. (10) is as fast as possible.

Inserting Eq. (10) into (8) and equating like powers in $\tau$ gives the recursion relation

$$
\begin{aligned}
(m+1) x_{k, m+1}= & \omega m x_{k, m} \\
+\sum_{i=0}^{\infty} k[ & G_{i} x_{k+i-1, m}+(k-1) D_{i} x_{k+i-2, m} \\
& \left.\quad+\sum_{j=0}^{m} F_{i} x_{1, j} x_{k+i-1, m-j}\right]
\end{aligned}
$$

with

$$
x_{k, 0}=\left\langle x^{k}(0)\right\rangle, \quad x_{0, m}=\delta_{0, m},
$$

where the last statement means the normalization condition. It is a simple matter to show that the conventional Taylor series expansion (5) follows from the above results in the linear case $F=0$ with $\omega=0(\tau=t)$. When applied to the mean-field model (1), Eq. (12) yields

$$
\begin{aligned}
(m+1) x_{k, m+1}= & {[\omega m+k(1-\theta)] x_{k, m} } \\
& -k x_{k+2, m}+k(k-1) D x_{k-2, m} \\
& +\sum_{j=0}^{m} k \theta x_{1, j} x_{k-1, m-j}
\end{aligned}
$$

It is thus seen that with this technique the treatment of the nonlinear problem (1) does not present a more difficult task than that of the conventional linear case $\theta=0$. The 
expansion coefficients are easily determined recursively in terms of Eq. (14) starting with $x_{k, 0}$.

The above expansion is formally exact and, therefore, independent of the choice of the free parameter $\omega$. As we are unable to handle the infinite expansion in Eq. (10), we have to truncate it at some finite $m=M$. Then, the resulting approximations $\left\langle x^{k}(t)\right\rangle_{M}$ of the moments depend on $\omega$. Unfortunately, establishing general convergence criteria for the expansion (10), which depend on the free parameter $\omega$, seems a quite difficult task. Since, however, the error introduced in $\left\langle x^{k}(t)\right\rangle$ due to the series truncation depends on $\omega$ and $t$, a rigorous way to minimize it is to consider $\omega$ as a function of $t$ and to optimize its time evolution as discussed in Ref. [13]. Here we employ a time-independent criterion for determining $\omega$ which is based on the true evolution equations for the moments. For example, if we are interested in the time evolution of the first moment of Eq. (1), one expects that a good approximation for this moment can be obtained by minimizing the error functional

$$
I(\omega)=\int_{0}^{T} d t\left(\partial_{t}\langle x\rangle-\langle x\rangle+\left\langle x^{3}\right\rangle\right)_{M}^{2},
$$

where $[0, T]$ is the interval in which an accurate description of $\langle x(t)\rangle$ is desirable, and where the subscript $M$ means that the moments in the parentheses are not the exact ones but their approximations obtained by using the series representation (10) truncated at $m=M$. The resulting equation is readily solved for $\omega$ by any root finding procedure. We note that the optimal values of $\omega$ turn out to be rather insensitive with respect to $T$. On the other hand, as the truncation error does not necessarily tend to zero with $t$ going to infinity, the upper integration limit in Eq. (15) has to be chosen finite, in order to avoid divergence. In our calculations we have taken $T=2$. The corresponding generalization of Eq. (15) to an arbitrary moment of the process $(6)$ reads

$$
\begin{aligned}
I(\omega)=\int_{0}^{T} d t[ & \partial_{t}\left\langle x^{k}\right\rangle-k\left\langle x^{k-1} G\right\rangle \\
& \left.-k\langle x\rangle\left\langle x^{k-1} F\right\rangle-k(k-1)\left\langle x^{k-2} D\right\rangle\right]_{M}^{2} .
\end{aligned}
$$

Minimizing the above functional provides one with a minimal average error of the moment $\left\langle x^{k}(t)\right\rangle_{M}$ in the interval $[0, T]$, and, consequently, a reasonable choice of the free parameter for a given power $k$ and truncation number $M$. In principle, a time-dependent criterion for minimizing the error at each time moment $t$, and, therefore, for optimizing the time evolution of $\omega(t)$ in the whole time domain can also be constructed, but we will not do so here.

In order to illustrate the power of the present technique we first consider a linear Fokker-Planck equation given by

$$
\partial_{t} P(x, t)=\partial_{x}\left(r x+D \partial_{x}\right) P(x, t) .
$$

Its fundamental solution is unique and has the form

$$
\begin{aligned}
P(x, t \mid y)= & {\left[2 \pi D\left(1-e^{-2 r t}\right) / r\right]^{-1 / 2} } \\
& \times \exp \left[-\frac{r\left(x-y e^{-r t}\right)^{2}}{2 D\left(1-e^{-2 r t}\right)}\right] .
\end{aligned}
$$

A straightforward application of the above formalism to Eq. (17) shows that the exact expressions for the moments are already obtained with just the first term of the expansion (10),

$$
\begin{aligned}
\langle x(t)\rangle & =y e^{-r t}, \\
\left\langle x^{2}(t)\right\rangle & =y^{2} e^{-2 r t}+\frac{D}{r}\left(1-e^{-2 r t}\right) .
\end{aligned}
$$

By contrast, infinitely many terms are to be included in the Taylor series (5) to obtain the same results [11].

As a second and more challenging example, we consider Eq. (1). This equation has been solved with the initial condition

$$
P(x, 0 \mid y)=\delta(x-y)
$$

for different initial values $y$ and for several points $(\theta, D)$ above the critical line. The numerically exact results have been calculated by means of a finite-difference method which will be published elsewhere [7]. The main findings are as follows. The present power series expansion converges rapidly over a broad range of $t$. It is particularly efficient in dealing with a situation when the system starts not too far from the stationary value of the first moment $\langle x(\infty)\rangle$. With an increasing difference $y-$ $\langle x(\infty)\rangle$ the convergence slowly becomes worse. What is especially pleasing is that the expansion turns out to produce surprisingly accurate results not only in the shortto-intermediate but also in the long time regime. Accurate values for the stationary moments $\left\langle x^{k}(\infty)\right\rangle$ can be obtained with just a few terms of the series in Eq. (10). These are found to be dependent of the initial condition $y$ showing all the peculiarities of the considered system. To the best of our knowledge, the present work is the first application of the power series expansion formalism to nonlinear problems.

As expected, the above results are in drastic contrast to those obtained with the Taylor series expansion of the moments in $t$. The latter follows from Eq. (10) for $\omega=$ 0 , and fails to converge starting already with $t \geq 0.5$ even though the system is initially located at $y=\langle x(\infty)\rangle$. Our findings are well illustrated in Fig. 1 which shows the relative error in the first moment $\langle x(t)\rangle$ made by truncating the series in Eq. (10) at $M=3,5$, and 7 as a function of $t$ for $D=0.1, \theta=2$, and $y=0.5$. Each successive higher order is seen to reduce the error over a larger range of $t$, and a very accurate description in the whole time domain is already achieved with $M=7$. In particular, the corresponding stationary value $\langle x(\infty)\rangle_{7}=$ 0.955 is in excellent agreement with the numerically exact 


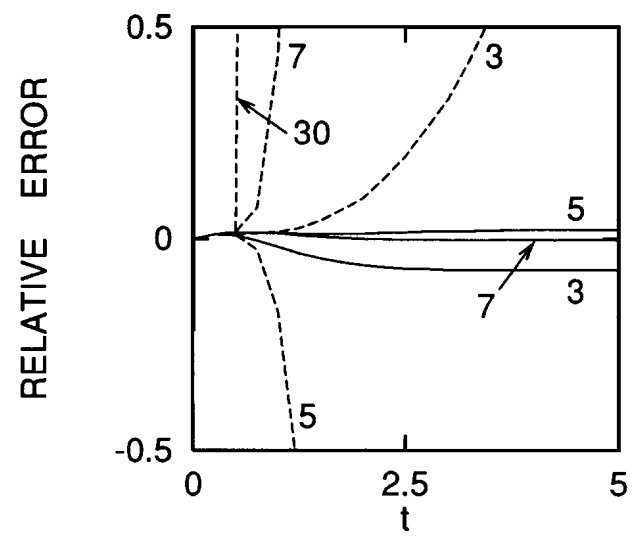

FIG. 1. Relative error, (approximate - exact)/exact, in the moment $\langle x(t)\rangle$ for a mean-field stochastic model [Eq. (1)] for $D=0.1, \theta=2$, and $y=0.5$. The solid lines are the results of the present power series expansion for $M=3(\omega=$ $1.43), 5(\omega=1.40)$, and $7(\omega=1.53)$, and the dashed lines are the results of the Taylor series expansion in the time $(\omega=0)$ for $M=3,5,7$, and 30 .

result $\langle x(\infty)\rangle=0.958$. We did not use a time-dependent criterion for determining the free parameter $\omega$ but expect a further improvement of the results especially for small $M$ 's in the intermediate-to-long time regime. Also shown are results obtained from the corresponding (i.e., of the same order in $t$ ) Taylor series expansion in the time. It is seen to diverge very rapidly with any finite number of terms taken into account, being thus of no practical importance.

In closing we would like to emphasize that the above examples are not exhaustive with respect to methodology. Besides the moments of the Fokker-Planck equation, the present power series expansion method can also be used for systematically treating various correlation function of the form

$$
\langle a(t) b(0)\rangle=\int_{-\infty}^{\infty} d x d y a(x) P(x, t \mid y) b(y)
$$

that play an important role in the theory of stochastic processes. It is a method that can be applied to simple or complex systems and neither nonlinearity nor high dimensionality seem to present special problems. We also note the relative ease with which this method can be implemented. Almost all calculations are doable analytically in a simple, economical way, thus allowing one to get very accurate results with a minimal computational ef- fort. From this point of view the present method is both theoretically and numerically advantageous with respect to various approximate Runge-Kutta algorithms that have been used so far to numerically integrate the hierarchy of equations for the moments $(8)[2,6]$.

Finally, a few remarks on the validity of the moment description itself (but not the power series expansion method) are in order. Recursion relations for the moments, Eq. (8), are known to be computationally efficient when dealing with systems that remain monostable for all $t$. In such a case, they converge rapidly, and neglecting moments of high enough order is known to be sufficient to get accurate results in the entire time domain. But they become ineffective and may even give divergent results for bistable systems [14], as well as for globally monostable systems, which demonstrate the transient bimodality for an initial period, provided this period is not too short [7].

We acknowledge the support of the Dirección General de Investigación Científica y Técnica of Spain for financial support (A.D.) and for Project No. PB92-0682 (M.M.).

*Permanent address: Institute for High Temperatures, 13/ 19 Izhorskaya Street, 127412, Moscow, Russia.

[1] K. Kometani and H. Shimizu, J. Stat. Phys. 13, 473 (1975).

[2] R. Desai and R. Zwanzig, J. Stat. Phys. 19, 473 (1978).

[3] D. Dawson, J. Stat. Phys. 31, 29 (1983).

[4] M. Shiino, Phys. Rev. A 36, 2393 (1987).

[5] A. N. Drozdov, Phys. Lett. A 171, 175 (1992); Physica (Amsterdam) 196A, 258 (1993).

[6] J. J. Brey, J. M. Casado, and M. Morillo, Physica (Amsterdam) 128A, 497 (1984).

[7] A. N. Drozdov and M. Morillo, Phys. Rev. E 54, 931 (1996).

[8] N. Makri and W. H. Miller, J. Chem. Phys. 90, 904 (1989).

[9] T. Miyazawa, Phys. Rev. A 39, 1447 (1989).

[10] A. N. Drozdov, Physica (Amsterdam) 196, 283 (1993); Z. Phys. B 90, 241 (1993).

[11] G. H. Weiss and M. Gitterman, Phys. Rev. E 51, 122 (1995).

[12] A. N. Drozdov, Phys. Rev. Lett. 75, 4342 (1995).

[13] A. N. Drozdov, J. Chem. Phys. 105, 515 (1996).

[14] I. I. Fedchenia, J. Phys. A 25, 6733 (1992). 